\title{
Influence of a hyperlipidic diet on the composition of the non- membrane lipid pool of red blood cells of male and female rats
}

Xavier Remesar, Arantxa Antelo, Clàudia Llivina, Emma Albà, Lourdes Berdié, Silvia Agnelli, Sofía Arriarán, José Antonio Fernández-López, Marià Alemany

Background and objectives. Red blood cells (RBC) are continuously exposed to oxidative agents, affecting their membrane lipid function. However, the amount of lipid in RBCs is higher than the lipids of the cell membrane, and includes triacylglycerols, which are no membrane components. We assumed that the extra lipids originated from lipoproteins attached to the cell surface, and we intended to analyse whether the size and composition of this lipid pool were affected by sex or diet.

Experimental design. Adult male and female Wistar rats were fed control or cafeteria diets. Packed blood cells and plasma lipids were extracted and analysed for fatty acids by methylation and GC-MS, taking care of not extracting membrane lipids.

Results. The absence of w3-PUFA in RBC extracts (but not in plasma) suggest that the lipids extracted were essentially those in the postulated lipid surface pool and not those in cell membrane. In cells' extracts, there was a marked depletion of PUFA (and, in general, of insaturation). Fatty acid patterns were similar for all groups studied, with limited effects of sex and no effects of diet in RBC (but not in plasma) fatty acids. Presence of trans fatty acids was small but higher in RBC lipids, and could not be justified by dietary sources. Conclusions. The presence of a small layer of lipid on the RBC surface may limit oxidative damage to the cell outer structures, and help explain its role in the transport of lipophilic compounds. However, there may be other, so far uncovered, additional functions for this lipid pool. 
1

2

3

4

5 Influence of a hyperlipidic diet on the composition of the non-membrane lipid 6 pool of red blood cells of male and female rats

7 Xavier Remesar ${ }^{1,2,4}$, Arantxa Antelo ${ }^{1,4}$, Clàudia Llivina ${ }^{1}$, Emma Albà $^{1}$, Lourdes Berdié3

8 Silvia Agnelli", Sofía Arriarán ${ }^{1}$, José Antonio Fernández-López ${ }^{1,2,4}$, Marià Alemany 1,2,4

9 10

1

12

13

14

15

16

17

18

VERSION 2, June 2015

4

1 Department of Nutrition and Food Science, Faculty of Biology, University of Barcelona,

10 Barcelona Spain

12 Institute of Biomedicine, University of Barcelona, Barcelona Spain

12 Scientific \& Technical Services, University of Barcelona, Barcelona Spain

${ }^{4}$ CIBER OBN, Barcelona Spain

5 Corresponding Author: Dr. Marià Alemany; Department of Nutrition and Food Science, Faculty 6 of Biology, University of Barcelona; Av. Diagonal, 643; 08028 Barcelona, Spain Tel. 17 34934034606; e-mail: malemany@ub.edu 


\section{Introduction}

Most of blood-borne lipids are carried by plasma lipoproteins, which play a critical role in the transfer and distribution of energy between organs and tissues. Red blood cells (RBC) integrity is essential, and largely depends on their external membranes; thus, there is abundant literature on RBC lipids, and their modulation by diet (Gibson et al. 1984; Rotimi et al. 2012; Volek et al. 2004) or disease (Ferreri et al. 2005; Labagambe et al. 2008; Lemaitre et al. 2002).

Analysis of RBC lipid classes showed the presence of different types of phospholipids, cholesterol (and its esters), but also significant amounts of triacylglycerols (TAG) (Rotimi et al. 2012; Volek et al. 2004). However, TAG are not components of cell membranes; mammal RBCs could not oxidize lipids, and thus are devoid of reserves. Consequently, the presence of TAG in RBC, widely demonstrated (Volek et al. 2004), remains unexplained, since mammalian RBCs do not contain intracellular membranous structures. The outstanding presence of TAG adds to the ample variability on the proportions of lipid classes (and fatty acids) found by different studies (Carlson et al. 1986; Rotimi et al. 2012) in RBC. These data, taken together, hint at the existence of lipid depots in RBCs that are different from (true) membrane lipids. As far as we know, however, in RBC no specific lipid stores have been described which could explain the presence of TAG and other lipid classes in excess of those forming part of cell membrane structure.

RBCs are in constant contact with plasma lipids (and lipophilic hormones); they have a direct relation with apolipoproteins (Cooper et al. 1977), bind lipoproteins (Hui et al. 1981), and have been found to carry free cholesterol to the liver (Hung et al. 2012). RBCs carry/harbour a number of steroid hormones (Romero et al. 2012), as well as extra glucose and amino acids loosely adsorbed on their surface (Proenza et al. 1994). This disposition has been suggested to speed up the interchange of substrates with the epithelial cells lining the capillary walls (Elwyn et al. 1972).

RBCs, membranes are in close proximity of those of endothelial cells, with cyclic changes in exposure (due to constant blood cycling) to different (tissue and systemic venous/arterial blood) $\mathrm{pH}$ and $\mathrm{pO}_{2}$. These changes compound the continuous variations in other plasma components (fatty acids, lipoproteins, cholesterol, glucose, etc.) and exposure to free radicals (superoxide, peroxynitrite, nitric oxide) in tissues (Kagota et al. 2009). Consequently, the structure and fatty acid composition of RBC membrane lipids change with their age, in spite of an active lipid turnover, affecting especially its external layer (Dise et al. 1980; Quarfordt \& Hilderman 1970), 
which composition is markedly different from the more stable inner layer (Zwaal \& Schroit 1997). In any case, constant exposure results in a relative loss of function, with increased rigidity, a condition aggravated in metabolic syndrome (van Blitterswijk et al. 1987).

Studies on the incorporation of labelled fatty acids showed a small but significant incorporation of them in RBCs in just $24 \mathrm{~h}$ (Leyton et al. 1987); this implies the existence of a rapid system for plasma fatty acid interchange with RBC lipids. This could not be fully explained by phospholipid or cholesterol turnover (Quarfordt \& Hilderman 1970; Reed et al. 1968), which are slower and related to median RBC half-life: 45-50 days in rats (Burwell et al. 1953). In addition, the substitution of internal membrane phospholipids is extremely slow (Zwaal \& Schroit 1997). We hypothesized that the rapid lipid interchange repeatedly observed in RBC may be related to a more accessible pool, in equilibrium with plasma, rather than to the necessarily stable cell membrane lipid bilayer.

A report suggest the presence of a fuzzy (probably lipid) cover on the RBC of hyperlipidemic blood when examined under the microscope (Miller et al. 2013), and the overall lipid content of packed blood cells is higher than what can be expected from its membrane lipids alone. We hypothesized that the ability of RBC to transport lipophilic compounds and the presence of non-membrane lipids may be a consequence of the more or less loose presence of lipoproteinderived lipids attached/bound to the surface of the RBC. This pool may participate in the turnover/repletion of RBC membrane lipid fatty acids. In order to test the hypothesis we analysed RBC lipids using a known mild lipid extraction procedure (i.e. assumedly not affecting their membrane lipids), comparing the fatty acid patterns and quantitative analysis of the lipids extracted in function of sex and exposure to a hyperlipidic diet.

\section{Materials \& Methods}

Animals, diets, and experimental setup

All animal handling procedures were carried out in accordance with the norms of European, Spanish and Catalan Governments. The Animal Ethics Committee of the University of Barcelona approved the specific procedures used.

Nine week old female and male Wistar rats (Harlan Laboratory Models, Sant Feliu de 
Codines, Spain) were used. Six animals per group were housed in two-rat cages, had free access to water. The animals were kept in a controlled environment (lights on from 08:00 to 20:00; 21.5$22.5^{\circ} \mathrm{C} ; 50-60 \%$ humidity). Two groups of animals for each sex were randomly selected and were fed ad libitum, for 30 days, with either normal rat chow (Harlan \#2014) or a simplified cafeteria $\operatorname{diet}$ (Ferrer-Lorente et al. 2005). This diet was made up by chow pellets, plain cookies, with liver pâté, bacon, whole milk with $300 \mathrm{~g} / \mathrm{L}$ sucrose and a mineral plus vitamin supplemens. We used the procedures for food intake estimation and analysis described previously (Prats et al. 1989). Diet composition was (expressed as energy content): carbohydrate 67\%, protein $20 \%$, and lipid $13 \%$ for controls; the mean composition of the cafeteria diet ingested was carbohydrate $47 \%$, protein $12 \%$ and lipid $41 \%$. This diet induced a significant increase in body fat and has been used for a long time in comparative studies on metabolic syndrome (Ferrer-Lorente et al. 2010; FerrerLorente et al. 2005; Romero et al. 2009)

The known composition and analysed fatty acid composition of the food items (including the control rat chow pellets) allowed us to estimate the energy and nutrient content of the diets consumed. Table 1 shows the amount of each food consumed per rat and day, as well as their nutrient energy equivalences of the four experimental groups ( $\mathrm{N}=6$ for each) female-control (FC), female-cafeteria (FK), male-control (MC) and male-cafeteria (MK).

\section{Sampling}

At the end of the experiment, the animals were anesthetized with isoflurane and immediately killed by exsanguination from the exposed aorta, using dry heparinized syringes. Blood was centrifuged $20 \mathrm{~min}$ at $2000 \mathrm{xg}$, at $2-4^{\circ} \mathrm{C}$. Plasma was frozen; the plasma-free packed cells were also frozen; all samples were kept at $-20^{\circ} \mathrm{C}$.

Packed cell volume was calculated from its weight and density (previously measured: 1.11 $\mathrm{g} / \mathrm{mL}$ ); the percentage ratio of this volume $v s$. that of blood gave us the haematocrit value (Hc). Under the conditions of centrifugation used, packed cells included a $9.4 \%$ of its volume as trapped plasma, calculated according to previously published data (Romero et al. 2012).

\section{Plasma general analytical procedures}

Plasma triacylglycerols and total cholesterol were measured using the Biosystems kits $\# 11828$, and \#11505, respectively. Plasma non-esterified fatty acids were estimated with kit NEFA-HR(2) (Wako, Neuss, Germany). 
Sample lipid extraction

Samples of $0.050 \mathrm{~mL}$ of just thawed plasma, or about $0.20 \mathrm{~g}$ of frozen packed cells, were suspended (and gently vortexed) in $10 \mathrm{~mL}$ of trichloromethane: methanol $(3: 1 \mathrm{v} / \mathrm{v})$ (Folch et al. 1957) in screw-cap tubes with Teflon liners. The samples were extracted for $24 \mathrm{~h}$ in rotary mixers at room temperature. Then $2 \mathrm{~mL}$ of $9 \mathrm{~g} / \mathrm{L} \mathrm{NaCl}$ in water were added, and the extraction was continued for 1 hour. The aqueous supernatants, and, eventually, interface protein, were discarded. The organic phase was carried to clean tubes and dried under a gentle stream of nitrogen at room temperature; the lipid residue was used for fatty acid derivatization. This extraction procedure was repeated using samples of $0.500 \mathrm{ml}$ of plasma and about $0.5 \mathrm{~g}$ of packed cells, but now the dry residue was carefully weighed in order to measure the total lipid extracted from the samples using this procedure.

Food samples were powdered under liquid nitrogen and extracted overnight with trichloromethane: methanol (3:1 v/v) (Folch et al. 1957) and the samples processed for fatty acid analysis using the same procedure described for tissue samples.

Fatty acid analysis

Lipid residues were used for methylation (Christie 1993). In short: they were suspended in $0.50 \mathrm{~mL}$ of $100 \mathrm{~g} / \mathrm{L}$ boron trifluoride in methanol (Fluka, Buchs, Switzerland) (i.e., $116 \mu \mathrm{mol} \mathrm{F}{ }_{3} \mathrm{~B}$ ), taking care to suspend and dissolve all residues. The tubes were left standing in the dark at $4^{\circ} \mathrm{C}$ for $12 \mathrm{~h}$, coarsely covered with aluminium foil. Later, $1 \mathrm{~mL}$ hexane (Panreac, Castellar del Vallès Barcelona, Spain) and $2 \mathrm{~mL}$ pure water were added; the mixture was vortexed, the tubes capped again and left in an orbital rotary mixer for $15 \mathrm{~min}$. The upper (aqueous) phase was transferred to another tube, which was again brought to dryness under a gentle stream of nitrogen. The residues were dissolved in $0.150 \mathrm{~mL}$ of HPLC-quality hexane (Panreac). The whole volume was then transferred to $0.200 \mathrm{~mL}$ Mandrel GLS inserts (BC Scientific, Miami, FL USA) within Agilent screw cap vials (Agilent, Santa Clara, CA USA) which had $8 \mathrm{~mm} \mathrm{PTFE/silicone} \mathrm{septa} \mathrm{(Soltec,}$ Bether, CT USA). The samples were kept tightly closed at $-20^{\circ} \mathrm{C}$ until measurement.

Samples were analysed with a CG-MS system (QP2010 Shimadzu, Kyoto Japan) using a SP-2560 Supelco (Supelco, Bellefonte, PA USA) column. The samples were run using, as standards, an extended methylated fatty acid mixture (Supelco FAME mix C4-C24). Calculations were done using the Shimadzu FASST for GC-MS program (version 2). 
The rates of recovery of lipids (and in particular fatty acids) were analysed with internal standards of bis-C17:0 diacylglycerol (Sigma) randomly added to a number of duplicate samples.

140

141

142

\section{Calculations}

The contribution of trapped plasma in packed blood cells to total lipids and to each individual FA measurement was calculated for each individual rat from their matching analyses of plasma and RBC-extracted lipids (Table 2).

The approximate amount of RBC membrane lipid was estimated from the mean rat cell volume, $69 \mu \mathrm{m}^{3}$ (69 fL) (Balazs et al. 1960), normal cell counts (7.2x10 $0^{6}$ cells $/ \mu \mathrm{L}$ of blood) (Balazs et al. 1960), corrected by the hematocrit value. The mean cell diameter $(6.7 \mu \mathrm{m})$ was that of the biconcaval RBC flattened disk. If we calculate the diameter of a sphere with the same volume than the actual RBC, we would obtain a smaller diameter, $5.08 \mu \mathrm{m}$, but the actual surface area of the $\mathrm{RBC}$ is higher than that of a sphere of the same volume. By comparing data on humans' RBCs (i.e. $90 \mathrm{fL}$ volume were equivalent to a surface of about $136 \mu \mathrm{m}^{2}$ (McLaren et al. 1987) we obtained diameters for volume- or surface-equivalent spheres of, respectively, 5.5 and $6.6 \mu \mathrm{m}$. That is, the real RBC surface was equivalent to that of a sphere with a volume about $20 \%$ higher than that obtained from the simple translation of the actual RBC volume to a sphere. Applying the same relationship to rat cells, the "sphere diameter" was increased $20 \%$ to obtain an estimation of RBC surface; this way, we obtained a probably better approximation to a sphere with the surface area of a rat $\mathrm{RBC}$ using a mean diameter of $6.1 \mu \mathrm{m}$. The corresponding mean individual surface area of a rat $\mathrm{RBC}$ would then be $117 \mu \mathrm{m}^{2}$. Since the thickness of a RBC bilayer membrane is in the range of 8-10 $\mathrm{nm}$ (Shkulipa 2006), we used a mean value of $9 \mathrm{~nm}$. These data allowed an estimation of the total volume of membrane (i.e. lipid bilayer) in a single RBC: surface area multiplied by the layer thickness, i.e. $117 \times 0.009=1.05 \mu \mathrm{m}^{3}$. Since the corrected haematocrit value was in the range of $43 \%$, in $1 \mathrm{ml}$ of packed cells there will be about $16.7 \times 10^{9}$ cells, and thus the lipid bilayer volume in $1 \mathrm{ml}$ of packed cells will be: $1.05 \times 16.7 \times 10^{9} \mu \mathrm{m}^{3}$, i.e. $17.5 \mu \mathrm{L}$. The density of lecithin (as representative membrane lipid) is $1.03 \mathrm{~g} / \mathrm{mL}$, thus, the weight of lipids in $1 \mathrm{ml}$ (i.e. $1.1 \mathrm{~g}$ ) of packed $\mathrm{RBC}$ would be in the range of $18.4 \mathrm{mg}(1.7 \% \mathrm{w} / \mathrm{w})$. This accounts for about half of the lipids estimated in packed RBC, a value concordant with the $3 \%$ of lipid contained in clotted animal blood, when analysed as food.

The normal composition of RBC phospholipid, being a main lipid component (Vayá et al. 
168

169

170

171

172

173

174

175

176

177

178

179

180

181

182

183

184

185

186

187

188

1993), is known (Cooper et al. 1977; Pöschl et al. 1999); consequently, the weight of fatty acids account for about $57 \%$ of the total membrane lipid. Taking oleic acid as "model" for molecular weight and abundance (an oleoyl residue has a molecular weight of 270), and applying this value and the proportion of fatty acids to the estimated weight of membrane lipid for packed RBC we obtain about $39 \mu \mathrm{mol}$ fatty acids per $\mathrm{g}$ of packed cells. This figure is an approximate estimation of the amount of lipid expected to find in packed RBC if their bilayer membrane were the only source of cell lipid.

\section{Statistical methods}

Statistical analyses were carried out with two- or three-way ANOVA comparisons, using the Statgraphics Centurion XVI program package (Statpoint Technologies, Warrengton VA USA).

\section{Results}

Plasma lipids

There were no significant differences in plasma triacylglycerols and packed cell volume between the four groups of rats studied (Table 2). However, total cholesterol was modified by sex and diet (higher for females and cafeteria diet). Non-esterified fatty acids were unaffected by diet, but females showed higher plasma levels than males. There were no clear relationships between the plasma lipid parameters. Table 2 also shows the proportion of lipid recovered from packed $\mathrm{RBC}$. There were no differences attributable to sex and diet, the data being remarkably uniform.

The patterns of distribution of individual fatty acids in plasma total-lipids were also similar between the sex/diet groups (Figure 1). There were only a few statistical differences between individual fatty acids between them. In plasma, exposure to a cafeteria diet resulted in significant differences in palmitoleic and oleic acids. Sex affected the levels of stearic, $\alpha$-linolenic, eicosadienoic, gondoic and heneicosanoic acids. The similitude of overall distribution pattern was repeated when fatty acids were grouped in their main classes: saturated (SFA), monounsaturated (MUFA), polyunsaturated (PUFA, $\omega-3$ and $\omega-6$ ) and trans (Table 3); SFA and PUFA (mostly $\omega$ 6) were predominant, there were only trace amounts of $\omega-3$ PUFA and trans fatty acids were practically absent. Only a few female rats treated with the cafeteria diet showed measurable levels of trans fatty acids. However, the grouped sums of fatty acids showed a significant effect of diet 
197

198

199

200

201

202

203

204

205

206

207

208

209

210

211

212

213

214

215

216

217

218

219

220

221

222

223

224

225

for MUFA and $\omega-6$ PUFA, and effects for sex in SAT fatty acids. Total plasma fatty acids (i.e. the sum of all individual fatty acids analysed, expressed as $\mathrm{mM}$ ) were $4.1 \pm 0.4$ (male control), $9.8 \pm 3.3$ (male cafeteria), $5.3 \pm 0.6$ (female control), and $9.2 \pm 3.3$ (female cafeteria); the differences were significant $(\mathrm{P}=0.029)$ for diet but not for sex.

\section{Red blood cell extractable lipids}

Figure 2 shows the individual fatty acid levels in packed cell extracts of the four groups of rats. The patterns were highly similar, with only small differences induced by diet (palmitic and heneicosanoic acids), and none due to the effect of sex. In addition, there was a lesser variety of fatty acids than in plasma, with lower levels of PUFAs, and a small but clear presence of trans fatty acids (essentially elaidic acid). When considering the classes of fatty acids (Table 3), all groups showed a similar pattern, with low $\omega-6$ PUFA, nil presence of $\omega-3$ PUFA and a token presence of trans fatty acids.

\section{Double bond distribution}

The differences between plasma and RBC fatty acids widened when the number of double bonds was computed. The lower proportion of PUFA in RBC compared with plasma, and the nil influence on this parameter of SFA, resulted in a proportion of double bonds in the range of 0.5 per fatty acid molecule in RBC versus almost 1.5 in plasma lipids (Figure 3). The proportion of double bonds in PUFA with respect to the total sum of double bonds showed the same pattern; in plasma, insaturation was mostly due to PUFA, but in RBC, the share of MUFA was much higher. Finally, the proportion of trans double bonds with respect to the sum of total double bonds was small but significantly higher in the RBC lipids of all experimental groups than in plasma, where trans fatty acids showed higher variability and lower values.

\section{Estimation of fatty acids intake}

Table 4 presents an estimation of the mean intake (in mmol/day) of dietary fatty acids for controls and cafeteria-fed rats. The data were calculated from the consumption of each type of food item per cage and day and the composition in fatty acids of the foods offered to the rats shown in Table 1.

The distribution of the different types of fatty acids in both standard chow and self-selected cafeteria diet were considerably different, both in proportions and (in part) variety, giving rise to 
226

227

228

229

230

231

232

233

234

235

236

237

238

239

240

241

242

243

244

245

246

247

248

249

250

251

252

253

254

255

widely marked differences for sex, significant for all fatty acids except for the short-chain capric, lauric and myristic acids. The cafeteria diet-fed rats ingested daily a much higher proportion (and variety) of fatty acids than controls ( $\mathrm{p}<0.0001$ for all fatty acids studied), and male rats ate more than females.

An analysis of the correlation between daily fatty acid intake $v s$. circulating plasma fatty acids showed that the only correlations observed (using all animals) were for oleic and gondoic acids ( $\mathrm{p}=0.026$ and $\mathrm{P}=0.046$, respectively), there were no correlations for any of the other fatty acids, and the significance was lost when analysing the four groups of animals separately.

\section{Discussion}

A critical aspect of the validity of the data presented here showing the existence of a secondary lipid pool in RBC, different from that constituted by membrane lipids, is the comparison of the methodology used and the fatty acids profiles. Those found were different from those expected from membrane composition as described by other authors (Cooper et al. 1977; Ferreri et al. 2005; Lemaitre et al. 2002).

The usual procedure for the estimation of blood cell-membrane fatty acids, followed almost universally is: A) Separation and washing of RBC in order to remove all traces of plasma lipids. B) Breakup of the cells and separation of a membrane fraction clean of non-membrane proteins, especially haemoglobin, but also structural fibres such as spectrin, C) Solvent extraction (twice in most cases) with a suitable solvent and extraction conditions and time (Rose \& Oklander 1965), the remaining lipid (bound to proteins) being usually discarded. D) Overall methylation, usually in strong acid to hydrolyse complex phospholipids. E) Analysis through GC or CG-MS. F) Presentation of the results as percentages of total fatty acids measured, since step B), and in part step C), could hardly be quantitative, and the effectiveness of step D) is often problematic.

Since we assumed that the most probable place for the RBC non-membrane-bilayer lipid "stores" (i.e. the site containing the TAG found in RBC lipid extracts) was the cell surface, we decided not to wash the cells and extrude the plasma by centrifugation (but taking care to limit cell breakage). We estimated the mass of trapped plasma, which allowed discounting its contribution to the total (mildly) extracted lipids of packed cells. Evidently, our intention was to keep the membranes as inaccessible as possible to our extraction method, and thus we used a 
256 procedure suitable for the extraction of all lipid classes (Folch et al. 1957) which has been found 257 inadequate/ ineffective for RBC membrane lipid extraction (Eder et al. 1993; Rose \& Oklander 258 1965).

The extracted lipids were methylated with a method sufficiently powerful to release methyl-fatty acids from most phospholipids (Christie 1993), but which, in turn, was not sufficiently strong for all classes of complex membrane phospholipids (Eder 1995); in addition, we used internal standards to check the effectiveness of methylation.

The results obtained suggest that the RBC lipid extract was not representative of membrane lipids. First because of the practical absence of critical PUFA membrane components (such as docosahexaenoic), even when compared with plasma. Second because of the impoverishment in double bonds, compared with plasma (which we assume was its origin), essentially at the expense of PUFA. There was, also, a relative abundance of SFA. Both factors reflected a situation extremely different from that found in membranes (Carlson et al. 1986; Lemaitre et al. 2002). In any case, these results do not preclude the possibility that an unknown proportion of RBC membrane lipids would be extracted with the procedure used. The trichloromethane: methanol method indeed can dissolve most lipids from complex matrices, including membranes, as in brain (Folch et al. 1951), but we did not break massively all cells, only compressed and snap frozen them, preserving in part their structure. In addition, we discounted the lipids of trapped plasma. Nevertheless, since the mass of lipid recovered from cells was similar to the calculated weight of membrane lipids, a higher share of PUFA should be detected if membrane lipids were extracted in a significant proportion. Contrary to that, the results obtained strongly suggest that membrane lipids were not extracted in a proportion high enough to allow us to detect their typical PUFA presence/ pattern.

Most studies on RBC lipids have been done first purifying cell membranes from washed cells' ghosts (Eder et al. 1993; Rose \& Oklander 1965; Vayá et al. 1993). Using these methods, PUFA levels were higher, in contrast with studies analyzing "erythrocyte lipids" (Labagambe et al. 2008; Rotimi et al. 2012; Volek et al. 2004). The differences between these approaches support our assumption that the RBC lipid we obtained represented largely non-membrane RBC lipids.

The quantitative importance of the extracted lipids was highly dependent on diet, but not 285 on sex, with a proportion of 0.26 to $0.89 \%$ w/wet RBC weight, i.e. $1-2 \%$ w/dry RBC weight. 
286 However, the generalized lack of correlation of dietary fatty acid intake and the levels found in 287 plasma lipids showed that these relationships are not straightforward and may be modulated by

288

289

290

291

292

293

294

295

296

297

298

299

300

301

302

303

304

305

306

307

308

309

310

311

312

313

314

315

316

overall energy (largely lipid/ glucose) metabolism and substrate plasma turnover.

The easy availability for extraction of these RBC lipids, together with their abundance in MUFA and SFA agree with a lipoprotein origin of the deposits and their placement on the RBC surface. The findings of fuzzy borders in direct microscopic examination of blood cells from hyperlipidic plasma (Cooper et al. 1975; Miller et al. 2013) may support this assumption. In addition, as far as we know, no internal RBC membrane or lipid depots have been described for mammals. However, the lack of significant effects of diet (hyperlipidic in our case) on the mass of recovered RBC lipids suggests that the lipid "cover" of RBC should be rather thin (it would be similar in thickness to the membrane lipid bilayer if it were spread uniformly) and not directly dependent on plasma lipid content. The lack of relationship seems to preclude the occasional weak bonding of lipoproteins as such, a possibility based on the presence of anchor proteins for apolipoproteins on the RBC surface (Hui et al. 1981). The thin layer (or discontinuous blobs) of lipid is bound, probably, rather permanently to the actual membrane, since its proportion of double bonds (compared with plasma), essentially from PUFA, and the small presence of trans fatty acids suggests a sustained exposure of these lipids to oxidizing or nitrating agents (at least to a higher degree than their plasma counterparts). In any case, these differences show that their turnover is slower than that of plasma lipids.

Nitric oxide is known to favour the conversion of cis to trans double bonds (Proell et al. 2002), lowering the fluidity of membranes and affecting their function. Oxidative attacks by superoxide and other free radicals tend to break down unsaturated fatty acids, mainly PUFA (Mattson \& Grundy 1985; Trotschansky \& Rubbo 2008), which levels tend to decrease in structures continuously exposed to oxidizing environments. This is in part corrected by turnover of membranes in cells, and by interchange with lipoproteins in RBC (Cooper et al. 1975; Dise et al. 1980; Quarfordt \& Hilderman 1970; Reed et al. 1968). However, the marked lack of PUFA (and absence of $\omega-3$ ) indicate that: A) The postulated outer layer of lipid in RBC should be rather permanent, at least enough to show the effects of oxidation and nitration on its fatty acids. B) This lipid is repeatedly exposed (for all its functional life) to highly oxidative microenvironments in capillary beds. C) The lipid occupies a limited and defined space on the cells, which is not directly affected by the availability (or turnover) of lipids in plasma. 
In any case, there must be a certain degree of interchange of lipids between the RBC outer

318 lipid layer and plasma lipids since its comparison with lipoprotein fatty acid patterns shows a considerable degree of similitude if PUFA are excluded. Labelled fatty acids are rapidly incorporated into RBC (Leyton et al. 1987), and interchange or reposition of PUFA in the outer layer of RBC membranes has been previously described (Dise et al. 1980; Reed et al. 1968). Furthermore, diets high in PUFA decrease the stiffness of RBC membranes in metabolic syndrome (Katan et al. 1997; Pöschl et al. 1999). Probably there is a direct relationship between these phenomena, and this can be a function, so far not defined, of the external lipid layer of RBC. We postulate that it may act as an intermediate step for repairs (or protection) of the RBC membrane, since in mammals most maintenance systems must be external to the RBC, because they lack nuclei, ribosomes and most of the cell turnover machinery.

We expected, at least in the rats with overweight, that as a consequence of the cyclic exposure and close contact of RBC with endothelia there would be a marked increase in trans fatty acids (Alemany 2012), a consequence of the higher production of nitric oxide and other oxidative and nitrating agents (Ghasemi et al. 2012). The levels of trans fatty acids we actually found were small, but could not be justified by the residual levels found in the diet $(<0.1 \mathrm{nmol} /$ day $)$. Endogenous production of trans fatty acids is linked to the production of nitric oxide by RBC themselves or by the neighbouring endothelial lining (Zambonin et al. 2006). In any case, PUFA are easily affected by oxidative and nitrative processes (Trotschansky \& Rubbo 2008), inducing membrane damages (van Blitterswijk et al. 1987). Perhaps, the presence of the outer RBC lipid layer may help the transfer (or interchange) of trans or damaged fatty acids (in exchange for "fresh" PUFA) to plasma lipid for disposal elsewhere, helping to extend the functionality of RBCs for a longer time.

In the end, the presence of this lipid pool results in a net loss of PUFA, probably a consequence of oxidative processes affecting first the lipid layer over the RBC, protecting the underlying membrane. The external lipid layer could, then, constitute a first line of defence against deleterious oxidative processes that shorten the lifespan and functionality of RBCs. In metabolic syndrome, RBC half-life is reduced, probably because of increased fragility and loss of flexibility (Kung et al. 2009). Increased dietary supply of PUFA tends to reduce the extent of this damage

347 foamy, etc.) and the external lipid pool of RBCs. 


\section{Conclusions}

We postulate the presence of a small lipid pool on the RBC surface. This layer may help minimize the effects of oxidative damage on RBC membranes, which affects the functionality and lifespan of RBCs, as shown by its marked deficit of PUFA. We speculate that the loss of PUFA is probably compensated through interchange with lipoproteins. Only residual trans fatty acids remained. The external lipid pool may, also help explain the role of RBCs in the transport of lipophilic compounds. The possible importance of the external lipid RBC layer should be analysed under the light of available information on the role of blood cells under conditions of hyperlipidemia and inflammation. So far, these questions have not been studied in depth, in spite of their potential importance in the transport of lipids and regulatory agents between organs and in the cell-to-cell interactions (transfer, signalling) within the tight space of tissue capillary lumen and its cell lining.

\section{Acknowledgements}

At the time this investigation took place, C. Llivina was a postgraduate student and E. Albà an undergraduate student.

\section{References}

367

368

369

370

371

372

373

374

375

376

Alemany M. 2012. Regulation of adipose tissue energy availability through blood flow control in the metabolic syndrome. Free Radical Biology and Medicine 52:2108-2119.

Balazs T, Grice HC, and Airth JM. 1960. On counting the blood cells of the rat with an electronic counter. Canadian Journal of Comparative Medicine and Veterinary Science 24:273-275.

Burwell EL, Brickley BA, and Finch CA. 1953. Erythrocyte life span in small animals. Comparison of two methods employing radioiron. American Journal of Physiology 172:718-724.

Carlson SE, Carver JD, and House SG. 1986. High fat diets varying in ratios of polyunsaturated to saturated fatty acid and linoleic to linolenic acid: A comparison of rat neural and red cell membrane phospholipids. Journal of Nutrition 116:718-725. 
377 378

379

380

381

382

383

384

385

386

387

388

389

390

391

392

393

394

395

396

397

398

399

400

401

402

403

404

405

406

Christie WW. 1993. Preparation of fatty acids for chromatographic analysis. Advances in Lipid Methodology 2:69-111.

Cooper RA, Arner E-C, Wiley JS, and Shattil SJ. 1975. Modification of red cell membrane structure by cholesterol-rich lipid dispersions. A model for the primary spur cell defect. Journal of Clinical Investigation 55:115-126.

Cooper RA, Durocher JR, and Leslie MH. 1977. Decreased fluidity of red cell membrane lipids in abetalipoproteinemia. Journal of Clinical Investigation 60:115-121.

Dise CA, Goodman DBP, and Rasmussen H. 1980. Definition of the pathway for membrane phospholipids fatty acid turnover in human erythrocytes. Journal of Lipid Research $21: 292-300$.

Eder K. 1995. Gas chromatographic analysis of fatty acid methyl esters. Journal of Chromatography 671B:113-131.

Eder K, Reichlmayr-Lais AM, and Kirchgeßner M. 1993. Studies on the extraction of phospholipids from erythrocyte membranes in the rat. Clinica Chimica Acta 219:93-104.

Elwyn DH, Launder WJ, Parikh HC, and Wise EM. 1972. Roles of plasma and erythrocytes in interorogan transport of amino acids in dogs. American Journal of Physiology 222:13331342.

Ferrer-Lorente R, Cabot C, Fernández-López JA, and Alemany M. 2010. Site-specific modulation of white adipose tissue lipid metabolism by oleoyl-estrone and/or rosiglitazone in overweight rats. Naunyn-Schmiedeberg's Archives of Pharmacology 381:339-348.

Ferrer-Lorente R, Cabot C, Fernández-López JA, Remesar X, and Alemany M. 2005. Effects of oleoyl-estrone with dexfenfluramine, sibutramine or phentermine on overweight rats. European Journal of Pharmacology 513:243-248.

Ferreri C, Angelini F, Chatgilialoglu C, Dellonte S, Moschese V, Rossi P, and Chini L. 2005. Trans fatty acids and atopic eczema/dermatitis syndrome: the relationship with a free radical cis-trans isomerization of membrane lipids. Lipids 40:661-667.

Folch J, Ascoli I, Lees M, Meath JA, and LeBaron FN. 1951. Preparation of lipide extracts from brain tissue. Journal of Biological Chemistry 191:833-841.

Folch J, Lees M, and Sloane-Stanley GH. 1957. A simple method for the isolation and purification of total lipides from animal tissues. Journal of Biological Chemistry 226:497-509. 
407 408

409

410

411

412

413

414

415

416

417

418

419

420

421

422

423

424

425

426

427

428

429

430

431

432

433

434

435

436

Ghasemi A, Zahediasl S, and Azizi F. 2012. High serum nitric oxide metabolites and incident metabolic syndrome. Scandinavian Journal of Clinical and Laboratory Investigation 72:523-530.

Gibson RA, McMurchie EJ, Charnock JS, and Kneebone GM. 1984. Homeostatic control of membrane fatty acid composition in the rat after dietary lipid treatment. Lipids 19:942-951.

Hui DY, Noel JG, and Harmony JA. 1981. Binding of low density lipoproteins to erythrocytes. Biochimica et Biophysica Acta 664:513-526.

Hung KT, Berisha SZ, Ritchey BM, Santore J, and Smith JD. 2012. Red blood cells play a role in reverse cholesterol transport. Arteriosclerosis Thrombosis and Vascular Biology 32:14601465.

Kagota S, Tada Y, Nejime N, Nakamura K, Kunitomo M, and Shinozuka K. 2009. Chronic production of peroxynitrite in the vascular wall impairs vasorelaxation function in SHR/NDmcr-cp rats, an animal model of metabolic syndrome. Journal of Pharmacological Sciences 109:556-564.

Katan MB, Deslypere JP, van Birgelen AP, Penders M, and Zegwaard M. 1997. Kinetics of the incorporation of dietary fatty acids into serum cholesteryl esters, erythrocyte membranes, and adipose tissue: an 18-month controlled study. Journal of Lipid Research 38:20122022.

Kung CM, Tseng ZL, and Wang HL. 2009. Erythrocyte fragility increases with level of glycosylated hemoglobin in type 2 diabetic patients. Clinical Hemorheology and Microcirculation 43:345-351.

Labagambe EK, Tsai MY, Hopkins PN, Ordovas JM, Peacock JM, Borecki IB, and Arnett DK. 2008. Erythrocyte fatty acid composition and the metabolic syndrome: A national heart, lung, and blood institute GOLDN study. Clinical Chemistry 54:154-162.

Lemaitre RN, King IB, Raghunathan TE, Pearce RM, Weinmann S, Knopp RH, Copass MK, Cobb LA, and Siscovick DS. 2002. Cell membrane trans-fatty acids and the risk of primary cardiac arrest. Circulation 105:697-701.

Leyton J, Drury PJ, and Crawford MA. 1987. Differential oxidation of saturated and unsaturated fatty acids in vivo in the rat. British Journal of Nutrition 57:383-393.

Mattson FH, and Grundy SM. 1985. Comparison of effects of dietary saturated, monounsaturated, 
and polyunsaturated fatty acids on plasma lipids and lipoproteins in man. Journal of Lipid Research 26:194-202.

McLaren CE, Brittenham GM, and Hasselblad V. 1987. Statistical and graphical evaluation of erythrocyte volume distributions. American Journal of Physiology 252:H857-H866.

Miller CE, Hirani B, and Bain BJ. 2013. Hyperlipidemia revealed by erythrocyte morphology. American Journal of Hematology 88:625-625.

Pöschl JMB, Paul K, Leichsenring M, Han SR, Pfisterer M, Bremer HJ, and Linderkamp O. 1999. Effects of dietary supplementation of saturated fatty acids and of n-6 or n-3 polyunsaturated fatty acids on plasma and red blood cell membrane phospholipids and deformability in weanling guinea pigs. Lipids 34:467-473.

Prats E, Monfar M, Iglesias R, Castellà J, and Alemany M. 1989. Energy intake of rats fed a cafeteria diet. Physiology and Behavior 45:263-272.

Proell JM, Mosley EE, Powell GL, and Jenkins TC. 2002. Isomerization of stable isotopically labeled elaidic acid to cis and trans monoenes by ruminal microbes. Journal of Lipid Research 43:2072-2076.

Proenza AM, Palou A, and Roca P. 1994. Amino acid distribution in human blood. A significant pool of amino acids is adsorbed onto blood cell membranes. Biochemistry and Molecular Biology International 34:971-982.

Quarfordt SH, and Hilderman HL. 1970. Quantitation of the in vitro free cholesterol exchange of human red cells and lipoproteins. Journal of Lipid Research 11:528-535.

Reed CF, Murphy M, and Roberts G. 1968. Phospholipid exchange between plasma and erythrocytes in man and the dog. Journal of Clinical Investigation 47:749-760.

Romero MM, Fernández-Lopez JA, Esteve M, and Alemany M. 2009. Site-related white adipose tissue lipid-handling response to oleoyl-estrone treatment in overweight male rats. European Journal of Nutrition 48:291-299.

Romero MM, Fernández-López JA, Remesar X, and Alemany M. 2012. Effect of sex and prior exposure to a cafeteria diet on the distribution of sex hormones between plasma and blood cells. PLoS One 7: e34381.

Rose H, and Oklander M. 1965. Improved procedure for the extraction of lipids from human erythrocytes. Journal of Lipid Research 6:428-431. 
Rotimi OA, Olayiwola IO, Ademuyiwa O, and Balogun EA. 2012. Effects of fibre-enriched diets on tissue lipid profiles of MSG obese rats. Food and Chemical Toxicology 50:4062-4067.

Shkulipa SA. 2006. Computer simulations of lipid bilayer dynamicsPhD thesis. University of Twente.

Trotschansky A, and Rubbo H. 2008. Nitrated fatty acids: Mechanisms of formation, chemical characterization, and biological properties. Free Radical Biology and Medicine 44:18871896.

van Blitterswijk WJ, van der Meer BW, and Hilkmann H. 1987. Quantitative contributions of cholesterol and the individual classes of phospholipids and their degree of fatty acyl (un)saturation to membrane fluidity measured by fluorescence polarization. Biochemistry 26:1746-1756.

Vayá A, Martínez M, Carmena R, and Aznar J. 1993. Red blood cell aggregation and primary hyperlipoproteinemia. Thrombosis Research 72:119-126.

Volek JS, Sharman MJ, Gomez AL, DiPasquale C, Roti M, Pumerantz A, and Kraemer WJ. 2004. Comparison of a very low-carbohydrate and low-fat diet on fasting lipids, LDL subclasses, insulin resistance, and postprandial lipemic responses in overweight women. Journal of the American College of Nutrition 23:177-184.

Zambonin L, Ferreri C, Cabrini L, Prata C, Chatgilialoglu C, and Landi L. 2006. Occurrence of trans fatty acids in rats fed a trans-free diet: A free radical-mediated formation? Free Radical Biology and Medicine 40:1549-1556.

Zwaal RFA, and Schroit AJ. 1997. Pathophysiologic implications of membrane phiospholipid assymetry in blood cells. Blood 89:1121-1132. 
1

Fatty acids present in plasma lipids of male and female rats, after exposure of 30 days to a hyperlipidic cafeteria diet, compared with controls.

The data are the mean \pm sem of 6 different animals per group.

Grey: SFA (saturated fatty acids); blue: MUFA (monounsaturated fatty acids); orange: $\omega-3$

PUFA (polyunsaturated fatty acids); red: $\omega-6$ PUFA; black: trans fatty acids.

Statistical significance of the differences between groups (2-way ANOVA); green star; $p<0.05$ for diet; purple diamond: $\mathrm{P}<0.05$ for sex. 

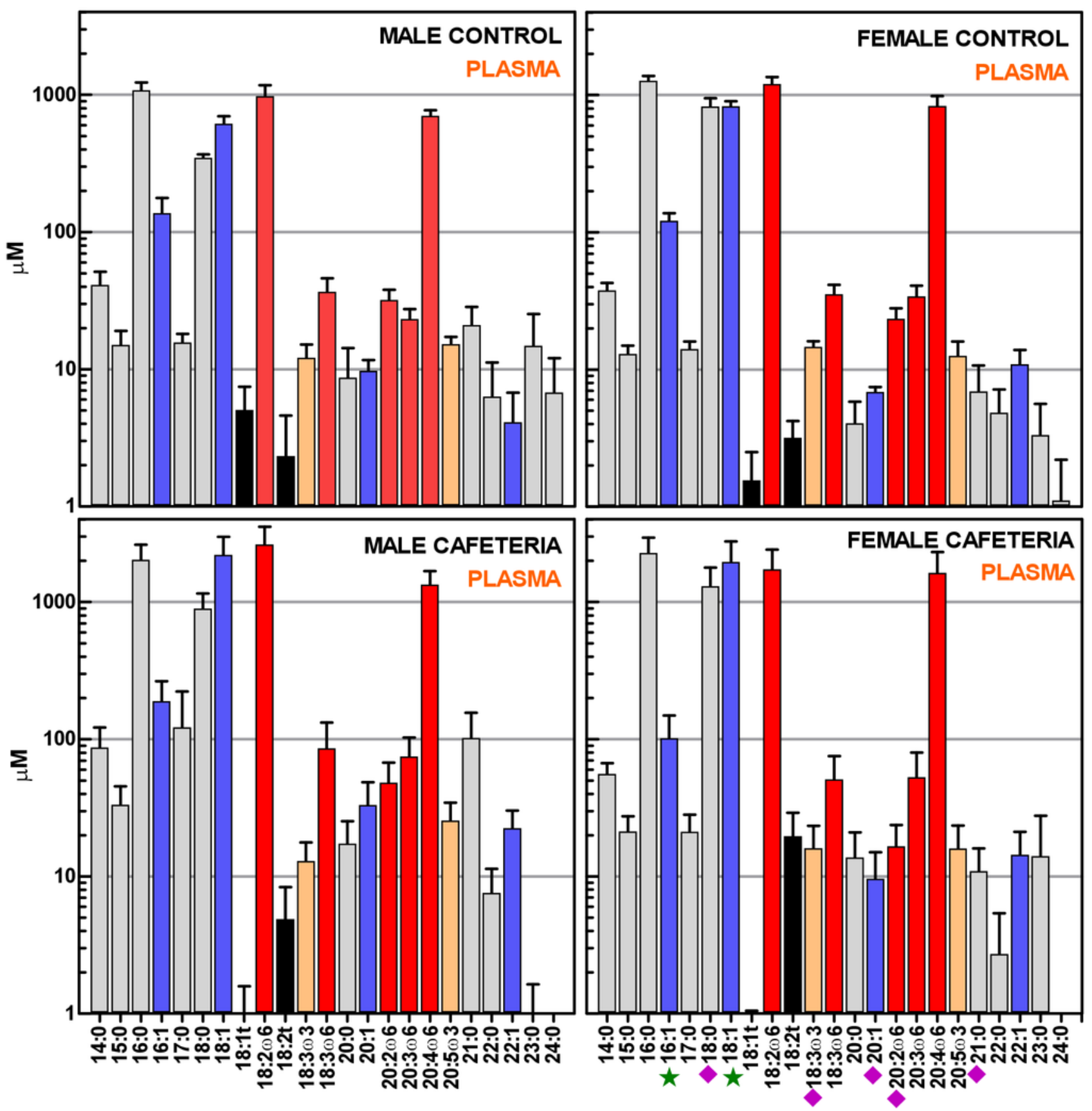
2

Fatty acids present in packed blood cell lipids of male and female rats, after exposure of 30 days to a hyperlipidic cafeteria diet, compared with controls.

The data are the mean \pm sem of 6 different animals per group, and are expressed in nmol/g of fresh cells.

Grey: SFA ; blue: MUFA ; red: $\omega-6$ PUFA; black: trans fatty acids.

Statistical significance of the differences between groups (2-way ANOVA); green star; $p<0.05$ for diet. 


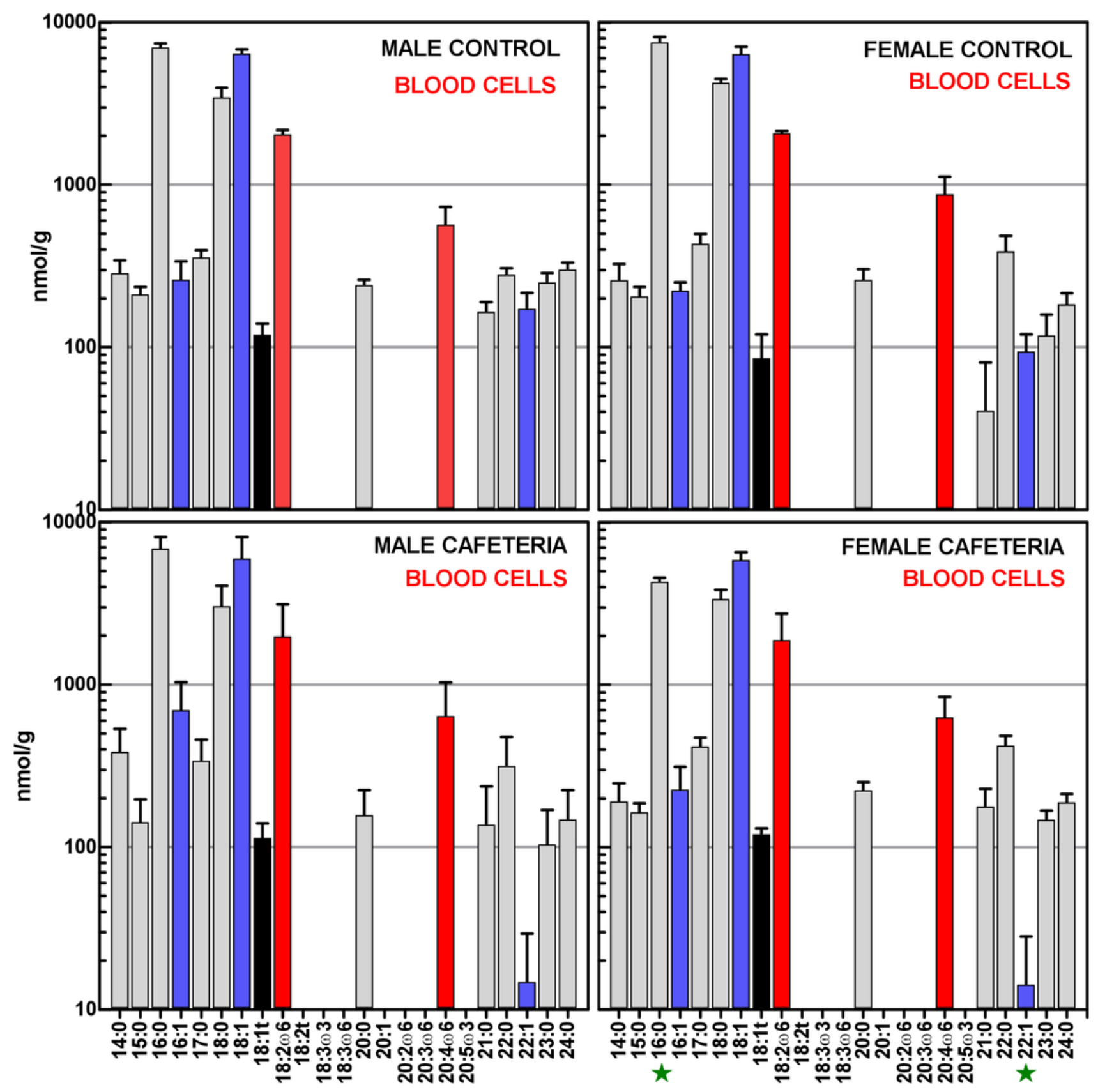




\section{3}

Distribution of double bonds in the lipids of RBC and plasma of male and female rats, during exposure of 30 days to a hyperlipidic cafeteria diet, compared with controls.

Left panel presents the mean number of double bonds per fatty acid molecule in RBC lipids (red columns) and plasma (pale yellow columns). Center panel depicts the percentage of trans fatty acid double bonds with respect to the total double bonds in RBC and plasma lipids. Right panel shows the percentage of double bonds that correspond to combined PUFAs in RBC and plasma lipids.

All data are the mean \pm sem of 6 animals per group. $\mathrm{M}-\mathrm{C}=$ male control; $\mathrm{F}-\mathrm{C}=$ female control; $\mathrm{M}-\mathrm{K}=$ male cafeteria, $\mathrm{F}-\mathrm{K}=$ female cafeteria. Statistical significance of the differences between compartments (cell vs. plasma lipids) calculated using a three-way ANOVA: a blue star indicates $P<0.05$. There were no significant differences related to sex or diet.
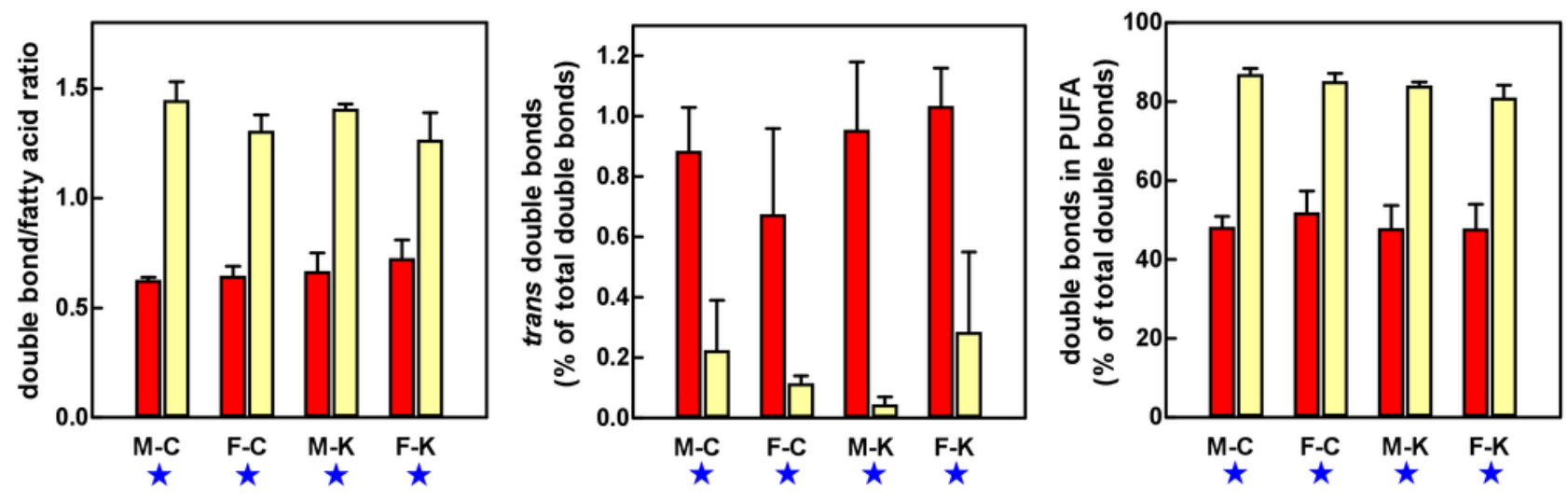


\section{Table $\mathbf{1}$ (on next page)}

Food intake of control and cafeteria diet-fed rats.

The data are the mean \pm sem of at least 20 days taken from three cages ( 2 rats in each) per group. Statistical significance of the differences between groups (2-way ANOVA): the columns represent the $P$ values for each variable: sex and diet. 


\begin{tabular}{|c|c|c|c|c|c|c|c|}
\hline & \multirow{2}{*}{ units } & \multicolumn{2}{|c|}{ male rats } & \multicolumn{2}{|c|}{ female rats } & \multicolumn{2}{|c|}{$P$ values } \\
\hline & & control & cafeteria & control & cafeteria & sex & diet \\
\hline \multicolumn{8}{|c|}{ NUTRIENT ENERGY INTAKE } \\
\hline total energy & $\mathrm{kJ} / \mathrm{d}$ & $272 \pm 15$ & $630 \pm 13$ & $209 \pm 13$ & $527 \pm 10$ & $<0.0001$ & $<0.0001$ \\
\hline carbohydrate & $\mathrm{kJ} / \mathrm{d}$ & $184 \pm 11$ & $312 \pm 4$ & $141 \pm 6$ & $274 \pm 9$ & $<0.0001$ & $<0.0001$ \\
\hline lipid & $\mathrm{kJ} / \mathrm{d}$ & $33.3 \pm 1.6$ & $259 \pm 4$ & $25.6 \pm 2.0$ & $210 \pm 10$ & $<0.0001$ & $<0.0001$ \\
\hline protein & $\mathrm{kJ} / \mathrm{d}$ & $54.8 \pm 2.3$ & $78.2 \pm 1.4$ & $40.1 \pm 3.1$ & $64.2 \pm 5.0$ & 0.0002 & $<0.0001$ \\
\hline \multicolumn{8}{|c|}{ FOOD ITEMS' INTAKE } \\
\hline rat chow & $g / d$ & $22.5 \pm 1.3$ & $5.53 \pm 0.45$ & $17.3 \pm 1.1$ & $4.95 \pm 0.95$ & 0.0079 & $<0.0001$ \\
\hline sugared milk & $\mathrm{mL} / \mathrm{d}$ & & $19.9 \pm 1.1$ & & $20.6 \pm 0.4$ & NS & $<0.0001$ \\
\hline plain cookies & $g / d$ & & $11.7 \pm 0.9$ & & $8.74 \pm 1.03$ & 0.0384 & $<0.0001$ \\
\hline bacon & $g / d$ & & $7.26 \pm 0.55$ & & $5.06 \pm 0.32$ & 0.0025 & $<0.0001$ \\
\hline pâté & $\mathrm{g} / \mathrm{d}$ & & $6.76 \pm 0.36$ & & $5.56 \pm 0.24$ & 0.0117 & $<0.0001$ \\
\hline
\end{tabular}


Table 2 (on next page)

Blood lipid distribution in female and male rats subjected 30 days to a cafeteria diet.

All values are the mean \pm sem of 6 different animals per group. Statistical analysis was done using a 2-way ANOVA. FA = fatty acids

${ }^{1}$ Data per gram of fresh packed cells; ${ }^{2}$ Percentage (in weight) of fatty acids in trapped plasma with respect to total fatty acids recovered from cells. 
Plasma total cholesterol

Plasma free fatty acids

Blood cell extracted lipid

FA extracted from RBC

FA in trapped plasma

Packed RBC volume

\begin{tabular}{ccccccc} 
& \multicolumn{2}{c}{ male rats } & \multicolumn{2}{c}{ female rats } & \multicolumn{2}{c}{ P values } \\
units & control & cafeteria & control & cafeteria & sex & diet \\
& $1.28 \pm 0.07$ & $1.40 \pm 0.11$ & $1.35 \pm 0.08$ & $1.48 \pm 0.07$ & $\mathrm{NS}$ & $\mathrm{NS}$ \\
$\mathrm{mM}$ & $1.76 \pm 0.11$ & $2.15 \pm 0.17$ & $2.25 \pm 0.21$ & $2.56 \pm 0.13$ & 0.0404 & 0.0106 \\
$\mathrm{mM}$ & $0.35 \pm 0.04$ & $0.43 \pm 0.03$ & $0.75 \pm 0.04$ & $0.68 \pm 0.08$ & $<0.0001$ & $\mathrm{NS}$ \\
$\mathrm{mM}$ & $12.0 \pm 3.5$ & $11.5 \pm 2.1$ & $12.8 \pm 3.2$ & $10.1 \pm 2.8$ & $\mathrm{NS}$ & $\mathrm{NS}$ \\
$\mathrm{mg} / \mathrm{g}^{1}$ & $21.9 \pm 0.4$ & $20.8 \pm 6.6$ & $23.2 \pm 1.9$ & $18.2 \pm 2.6$ & $\mathrm{NS}$ & $\mathrm{NS}$ \\
$\mu \mathrm{mol} / \mathrm{g}^{1}$ & $0.37 \pm 0.04$ & $0.88 \pm 0.29$ & $0.68 \pm 0.08$ & $0.82 \pm 0.30$ & $\mathrm{NS}$ & $\mathrm{NS}$ \\
$\mu \mathrm{mol} / \mathrm{g}^{1}$ & $1.7 \pm 0.2$ & $3.6 \pm 1.6$ & $3.0 \pm 0.4$ & $5.5 \pm 1.8$ & $\mathrm{NS}$ & $\mathrm{NS}$ \\
$\%{ }^{2}$ & $41.3 \pm 0.6$ & $44.1 \pm 1.6$ & $40.9 \pm 0.9$ & $42.1 \pm 0.6$ & $\mathrm{NS}$ & $\mathrm{NS}$ \\
$\%$ & & & & & &
\end{tabular}




\section{Table 3(on next page)}

Sum of SFA, MUFA, PUFA and trans-FA levels in plasma and blood lipids of male and female rats subjected to a control or a cafeteria diet for 30 days.

All values are the mean \pm sem of 6 different animals per group. Statistical analysis was done using a 2-way ANOVA. FA = fatty acids. 


\begin{tabular}{|c|c|c|c|c|c|c|c|}
\hline & \multirow{2}{*}{ units } & \multicolumn{2}{|c|}{ male rats } & \multicolumn{2}{|c|}{ female rats } & \multicolumn{2}{|c|}{$P$ values } \\
\hline & & control & cafeteria & control & cafeteria & sex & diet \\
\hline \multicolumn{8}{|l|}{ PLASMA } \\
\hline SFA & $\mathrm{mM}$ & $1.53 \pm 0.17$ & $3.25 \pm 1.02$ & $2.16 \pm 0.25$ & $3.67 \pm 1.18$ & 0.0300 & NS \\
\hline MUFA & $\mathrm{mM}$ & $0.76 \pm 0.12$ & $2.42 \pm 0.91$ & $0.96 \pm 0.10$ & $2.05 \pm 0.88$ & NS & 0.0235 \\
\hline$\omega-3$ PUFA & $\mathrm{mM}$ & $0.027 \pm 0.005$ & $0.038 \pm 0.014$ & $0.027 \pm 0.005$ & $0.032 \pm 0.014$ & NS & NS \\
\hline$\omega-6$ PUFA & $\mathrm{mM}$ & $1.75 \pm 0.24$ & $4.12 \pm 1.35$ & $2.11 \pm 0.31$ & $3.43 \pm 1.32$ & NS & 0.0435 \\
\hline trans-FA & $\mathrm{mM}$ & $0.007 \pm 0.004$ & $0.006 \pm 0.004$ & $0.005 \pm 0.001$ & $0.014 \pm 0.008$ & NS & NS \\
\hline \multicolumn{8}{|l|}{$\mathrm{RBC}$} \\
\hline SFA & $\mu \mathrm{mol} / \mathrm{g}$ & $12.43 \pm 0.77$ & $11.52 \pm 3.22$ & $13.54 \pm 1.25$ & $9.52 \pm 0.61$ & NS & NS \\
\hline MUFA & $\mu \mathrm{mol} / \mathrm{g}$ & $6.78 \pm 0.47$ & $6.60 \pm 2.49$ & $6,62 \pm 0.79$ & $6.04 \pm 0.83$ & NS & NS \\
\hline$\omega-3$ PUFA & $\mu \mathrm{mol} / \mathrm{g}$ & 0.00 & 0.00 & 0.00 & 0.00 & & \\
\hline$\omega-6$ PUFA & $\mu \mathrm{mol} / \mathrm{g}$ & $2.58 \pm 0.08$ & $2.59 \pm 1.25$ & $2.92 \pm 0.28$ & $2.50 \pm 1.08$ & NS & NS \\
\hline trans-FA & $\mu \mathrm{mol} / \mathrm{g}$ & $0.12 \pm 0.02$ & $0.11 \pm 0.03$ & $0.08 \pm 0.03$ & $0.12 \pm 0.01$ & NS & NS \\
\hline
\end{tabular}




\section{Table 4(on next page)}

Fatty acid intake of control and cafeteria-fed diet rats.

The data were calculated from the mean fatty acid composition of all foods given to the rats and the data of consumption indicated in Table 1. Values are the mean \pm sem. Statistical significance of the differences between groups (2-way ANOVA): the columns represent the $P$ values for each variable: sex and diet. 
fatty acid

$\mathrm{mmol} /$ day

capric

lauric

myristic

palmitic

stearic

arachic

behenic

lignoceric

palmitoleic

oleic

elaidic

gondoic

erucic

linoleic

linolenic

arachidonic male rats

control

$<0.1$

$<0.1$

$0.03 \pm 0.0$

$0.44 \pm 0.02$

$0.08 \pm 0.00$

$0.12 \pm 0.00$

$0.16 \pm 0.00$

$<0.1$

$<0.1$

$0.56 \pm 0.02$

$<0.1$

$0.11 \pm 0.0$

$<0.1$

$1.61 \pm 0.06$

$0.08 \pm 0.00$

$<0.1$

\begin{abstract}
cafeteria
\end{abstract}
$0.52 \pm 0.03$

$2.66 \pm 0.22$

$1.12 \pm 0.07$

$5.32 \pm 0.21$

$1.94 \pm 0.09$

$0.23 \pm 0.00$

$0.35 \pm 0.00$

$0.43 \pm 0.02$

$0.42 \pm 0.02$

$6.99 \pm 0.27$

$<0.1$

$0.25 \pm 0.0$

$0.15 \pm 0.1$

$2.15 \pm 0.04$

$0.20 \pm 0.00$

$0.14 \pm 0.01$ female rats

\begin{tabular}{cc}
\multicolumn{2}{c}{ female rats } \\
control & cafeteria \\
$<0.1$ & $0.43 \pm 0.04$ \\
$<0.1$ & $2.20 \pm 0.21$ \\
$0.02 \pm 0.0$ & $0.93 \pm 0.07$ \\
$0.34 \pm 0.01$ & $4.17 \pm 0.13$ \\
$0.06 \pm 0.00$ & $1.54 \pm 0.06$ \\
$0.09 \pm 0.00$ & $0.18 \pm 0.00$ \\
$0.13 \pm 0.00$ & $0.27 \pm 0.00$ \\
$<0.1$ & $0.34 \pm 0.01$ \\
$<0.1$ & $0.32 \pm 0.02$ \\
$0.42 \pm 0.02$ & $3.38 \pm 0.16$ \\
$<0.1$ & $<0.1$ \\
$0.09 \pm 0.0$ & $0.19 \pm 0.0$ \\
$<0.1$ & $0.12 \pm 0.01$ \\
$1.24 \pm 0.05$ & $1.63 \pm 0.02$ \\
$0.06 \pm 0.00$ & $0.15 \pm 0.00$ \\
$<0.1$ & $0.11 \pm 0.00$
\end{tabular}

\begin{tabular}{cc}
\multicolumn{2}{c}{$P$ P values } \\
sex & diet \\
NS & $<0.0001$ \\
NS & $<0.0001$ \\
NS & $<0.0001$ \\
$<0.0001$ & $<0.0001$ \\
0.0009 & $<0.0001$ \\
$<0.0001$ & $<0.0001$ \\
$<0.0001$ & $<0.0001$ \\
$<0.0001$ & $<0.0001$ \\
$<0.0001$ & $<0.0001$ \\
$<0.0001$ & $<0.0001$ \\
-- & -- \\
$<0.0001$ & $<0.0001$ \\
$<0.0001$ & $<0.0001$ \\
$<0.0001$ & $<0.0001$ \\
$<0.0001$ & $<0.0001$ \\
$<0.0001$ & $<0.0001$
\end{tabular}

